\title{
Indice de Documentos referentes al Juicio sobre legitima descendencia del último Inca Túpac Amaru
}

(Papeles existentes en la Unversidad Nacional de San Antonio del Cusco)

\section{INTRODUCCION}

En la Universidad Nacional de Sun Antonio del Cusco exister 12 lomos de documentos, pertenecientes a los siglos XVI.XIX, cuya común finelidad es la de probar que doña María Gertrudis de Avendaño Betanour y Túpac Amaru descendia cn línea recta del úlumo inca Túpac Arantu, condenado por el virrey Tolnto en la sequncta mítad del siglo XVI y contradecir as petensiones del crecique don José Gabriel Túpac Amaru.

Los 11 primeros tomos fuercn recopilados, en la segunda milaki del siglo XVIII, por don Vicente José García exposo de doña María Gertrudis. Ll lomo núrnero 12 eslá consliluido por dos piezas judiciales, dadas a pedimento de deña Crici?ia Guevara y Caslilla $y$ de don Manuel Sixto Lara, al comenzát la sequntio milaj del siglo XIX. Dichos 12 tomos har. sido reunidos en dos grupos: A y B.

GRUPO A.- Este grupo comprende cuatro tomos, bajo el rubro genérico de "Genealogía de D. Diego Sairitupac y Estados de Oropesa", alnque es de anolar que en el título del tomo I se omile la palabra "Diego". En general, el formalo cle los tomos de este grupo es de $33 \times 22$, y lodos están rubricados por don Vicente Iosé García.

El tomo I consta de 694 fojas de lexto y 10 hojas do indice, estando fechado on Lima a 5.XI-7@o. Además, llevis una nola, fechada en Madrid à 27.IX-794. El lomo II consla de 542 fojas de texto y 35 de indlce. estando fechado en Máirid a $28-V i-795$. El lomo III consta de 1093 fojas de texto y 10 fojas de indice, eslando fechado en Madrid a 28.VI-795. Y, finalmente, el lomo IV consla de 666 lojas de texto y 13 lojas de indice, estando fechado en Madrid a 28-VI-795.

GRUPO B.- Este grupo comprende acho tomos. El tomo I consta de 854 fojas de lexto y 13 fojas de indice, mostrando 72 instrumentos, contituidos por papeles do los st. 
glos XVI-XVIII. El tomo II consta de 635 fojas de texto y 10 fojas de índice, con papeles correspondientes a los sig̣los XVI-XVIII, mostrando un total de 40 instrumentos. El tomo III consta de 451 fojas, con 17 cuadernos, en lo civil y en lo criminal, y otras piezas con papeles correspondientes a los siglos XVI-XVIII. El tomo IV consta de 244 fojas de texto y cuatro foias de indice, con papeles correspondientes a los siglos XVI-XVIII, siendo un "Discurso Legal". Político, Jurídico, Histórico, y Genealógico" a cerca de la causa judicial antedicha. El tomo V consta de 239 fojas de texto y cuatro de índice, mostrando 14 instrumentos, destinados a señalar la filiación, ascendencia legítima y nobleza del teniente coronel don Vicente José García y Rodríguez, papeles correspondientes a los sigios XVI-XVIII, El tomo VI consta de 91 fojas y está dedicado a' los Indices de los cuatro tomos correspondientes al Grupo A. El tomo VII es la CERTIFICACION DE ARMAS, quo consta de 128 fojas. Es un libro impreso, cuyo texto fué reproducido en la Revista de la Universidad del Cusco, año XXII, segunda época, no 65, segundo semestre de 1933, p. 31-102. Finalmente, el tomo VIII consta de dos cuadernillos y 45 fojas, correspondientes a los primeros años de la segunda mitad del siglo XIX. El documento inicial tiene como fecha el 2-IV-859 y el documento final tiene como fecha el 23-X-860.

A continuación se inserta un Indice de los documentos relativos a los ocho tomos que forman el Grupo B, y que directamente interesan a la genealogía del cacique Túpac Amaru y a sus litigios judiciales que precedieron al levantamiento. Un detenido estudio de estos papeles explica porqué la justicia real falló a favor de los Betancur, después de la rebelión, habiendo estado hasta 1780 el litigio judicial predominantemente desfavorable a' dicha familia.

Daniel Valcárcel.

\section{T. I. Tamaño: $32 \times 20$}

\section{BibliogRupq Ẻe Letras}

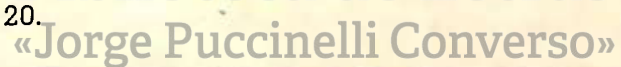

GEneAlogia / Le la Casa, y Familia de / Dn. Diego Felipe de Betancur / y Túpac Amaro y Hurtado de Arbieto./ Desde/ La feliz conquista y Pacificacion del / Reyno del Perú hasta el $\Lambda$ ño de 1790 / Libro 19 que / comprehende sesenta y dos Instrumentos, de $\mathrm{n}$ \% l hasta / el setenta $\mathrm{y}$ dos inclusive, en foxas 854. / Formado./ con el Libro $2^{\circ}$ (1) que se compone de Quarenta Documentos en foxas 635./ Por/ Dn. Vicente Jose Garcia Capitan Gra / duado de los Rs. Exercitos, Tente. Coronel/ y Comte del 4 \% Esquadrón del Reximto/ de Dragones de Cotabambas.

NUMERO 1\%/ Capítulos Impresos de la Hista. Pontificia, o Eccca./ Comprehenden/ la vida de Adriano Sexto Pontifice Maximo/ de la Iglesia de Dios el 218, exaltado a la/ Suprema Silla el año de 1492./ Y también/ El descubrimiento y conquista del Perú hasta/ la muerte de su sconquistadores Dn. Franco. Pizarro/ que falleció á manos de la violencia en 24 de Junio/ de 1541, por la muerte que hizo dar á Dn. Diego de/ Almagro el año antecedente, y siguen las epocas hasta 9 de Abril de 1548, en que ajusticlarón de Orden/ del Lizdo. Pedro de la Gasca á Franco. de Carbajal/ y Gonzalo Pizarro, con otros capitanes Reveldes/ en la Pampa de Anta que se llamó primero/ Jaquijaguana. (f. 1-9).

(1) Este libro constituye el tomo II (Grupo B). 


\section{ILUSTRACIONES: Papa Adriano VI.}

NUMERO 2\%/ REAL CONTSATA./ Celebrada por la Catholica Magestad de la Sra./ Da. Juana Reyna de Castilla, y de Leon, Madre! del Invizto Dn. Carlos 59 emperador de los Ro- / manos, y Augusto 19 Rey de las Españas; en la / ciudad de Toledo á 26. de Julio de 1529./ con/ El Marqués Dn. Franco. Pizarro por si, y sus/ compañeros Dn. Fernando de Luque Maestre Esquela/ y Provisor en sede vacante de la Sta. Yglesia del Da-/rien (que hoy está en Panamá) y Dn. Diego de / Almaçro vecino de la Ciudad de Panamá./ Para/ La Conquista, Pacificación y población del Rno./ del Perú, bajo las condiciones que expresa./ Nota/ Por la condición 16 de la Sta. Real Contrata $n^{\circ} 2$ que se celebró/ para la conquista del Perú se hizo Caballeros Ydalgos a los/ que no lo eran. y a los que tenían dicha calidad se les hizo/ Caballeros de la Espuela doradä; cuya Ydalguía se confirmó/ por R. a Cedula de 3 de IJulio de 573 (f. 10-13).

\section{ILUSTRACIONES: don Francisco Pizarro.}

NUMERO 3\%/ RESUMEN HISTORICO/ Del origen y succession de los Yncas, y demas/ soberanos del Perú./ con noticias/ De los successos mas notables en el Reyna do/ de cada uno./ PRIMER YNCA/ Fundador del Imperio, Manco Capac. (fs. 14-111). De Manco Cápac hasta Huáscar. p. 1-61 (fs. 14-44).

ANOTACIONES MARGINALES f, 14 (p. 1): "No es Lima, / la Capital del Perú,/ y si lo es la gran/ Ciudad del Cuzco, en/ virtud de muchos/ Rs. cedulas expedi-/ das desde el Sor./ Carlos 5. hta. nros./ tiempos qe. estan/ en los Libros de el/ Cavildo del Cuzco/ que he leido repeti / das veces- (f. 14 v. (p. 2): "Garcilazo es/ autor que. puede/ ser adiccionado/ en todas sus ptes." (Hablando de éste, como autor "el mas seguro").

F. 18 (p. 9): "Este Jemple esthoyileelonventolaelSton Domingo del Cuzco/ /Esta cosa es hoy/ el Convento de Re-/ligiosas de Sta. Cata/lina, del Orn. de Sto./Domingo: en el Cuzco "(Hablando del templo del Sol).

F. 38 (p. 50): (refiriendose a Túpac Yupanqui): "Tupac, significa/ el que resplandece./ Amaru, es cu-/lebra grande que /ay en los Andes, y / juntas las dos / dicciones dicen / Culebra resplan/deciente".

Desde Carlos I y Fco. Pizatro Hasta Fernando VI y Manso de Velasco. f.44 v. 105 (p. 62-184).

ANOTACIONES MARGINALES: F. 63 (f. 45 (refiriéndose al contrato de los 3 socios): "La compañia se / firmó el año de / 1525".

P. 64 (f. 45 v.) (refiriéndose a la capitulación de Toledo): "este despa-/ que tengo en/ mi poder, se sirmó / en Toledo a 26 de/ Junio de/ 1529".

P. 65 (f. 46) (españoles en el Cuzco) "Los primeros españoles / que pisarón el Cuzco / fueron Ernando de/ Soto y Pedro de / Varco". (la.).

P. 105 (f. 66) (hablando del nacimiento de Felipe II, en 1527) "En este año de/1527. nacio dn. / Juan Tito Tupac Amaro del Inga Tupac A-/maro qn. tomó el / nre. de Felipe el / año de 1542 que/ hizo la Capitu/lación con el Lizdo./ Vaca de Castro/ i quando

(1a.) Véase "Las Relaciones Primitivas de la Conquista del Perú" por Raúl Porras. p. 92, nota 53. 
su Abue/la doña Catalina/ Tucpe y Chilque/ mujer legitima de Manco Inga el $2 \%$ se bautizo el año 1534".

P. 62 (f. 69 v.) (refiriéndose a los hijos de Túpac Amaru) "fuerón 32 hijos/ y pariente de este / Inca segn. la Rl. Ceda./ qe reserbo".

Indice de las cosas notables contenidas en el este resumen: p. 185-195 (f. 106-111).

NUMERO 4./ ARBOLES GENEALCGICOS.- / ó / Demostración General/ DE / Los Yncas, ó Reyes, Señores Naturales,/ que en la Gentilidad Gobernarón las Pro-vincias del Reyno del Perú/ según Resulta de las Historias, varios Do-/cumentos, y Cedulas Reales de Nuestros / Catholicos Soberanos de la gloriosa memoria, que/ tan dignamente adquirierón este nuebo Reyno/ para la exaltación de nuestra Santa Feé./ ACOMPAÑ uno formado por mi, desdo el Inga Dn. Felipe Tupac-Amaro/ hasta dn. Diego Felipe Betancur su Quarto Nieto, con arreglo/ a los Documentos de su casa" (f. 112-117).

ILUSTRACIONES: Manco Inca.- Reyes españoles desde Carlos I hasta Luis I y Fe lipe V.- También aparece Atakualpa.- Arbol genealógico desde el inca Túpac Amaru hasta doña Gertrudis de Abendaño y Betancur.

NUMERO 5 CERTIFICACION ORIGINAL; Dada por Dn. Franco. Cosio. Teniente de Cura/ de la San!a Yglesia Cathedral de la Ciudad de los/ Reyes de las Pronvincias del Perú, en 20 de noviembre de 1779/ De/ La Partida de Bautismo hecho en 4 de sepre./ de 1538 en la persona de doña Ana Guainacapac,/ hija de Guainacapac, Inga que fué del Perú./ La que/ Se ha sacado del Libro que refiere para probar que/ incontinentí que los españoles llegaron al Perú, se / redujeron a la ovediencia de S. M. C., Y Gremio/ de Nuestra Santa Madre Yglesia, los hijos./ Nietos, yl Descendientes que al tiempo de la Con/quista existíandeles los Incas, que fueron de estos/Dominios, y que el no hallarse en el Cuzco Libros/de Bautismo de aquel liempo no puede obstar a los/ Ynteresados descendientes de dichos yncas"i. (f.118-121). ConversO»

\section{PARTIDA}

\section{Doña Ana/}

Huaina Ca-/

pac

(f. 119)

Hoy miércoles en Quatro (2) de/ septiembre de mil quinientos treinta y ocho años Bautizo el padre Labrador a doña Ana, hija de Huayna Caba, fueron Padrinos Hernando de Avila, y Antonio de Quiroz, madrina Catalina Ruiz mujer de Palencia Escrivano $=$ Diego Sánchez $=$.

Certificación del escribano de S. M. Pedro Lumbreras (fdo.) Torres.

Certificación de los escribanos: José Maraval, Teodoro Aillon Salazar, Valentin de

NUMREO 6/ REAL CEDULA/ Expedida por la Magestad del Sor. Emeprador/ Dn. Carlos $5{ }^{\circ}$ en 19 de Octubre de 1544./ A fabor/ De Dn. Juan Tito Tupac-Amaro Ynga hi-

(2) El día está escrito con tinta diferente. 
jo de Dn./ Felipe Tupac-Amàro Sor. que fué de las Provincios/ del Perú./ PUR LA QUE/ Lexitimó Su Majestad los hijos naturales cue tenía/ y concedió a dicho Dn. juan. a sus hijos, y Des/cendientes lexitimos el privilegio de usar/ de sus Reales Armas con una cadena en las / Puertas de su Casa, y Reporteros con las demas/ Mercedes que contiene (f. 122-127).

(fdo.) Juan de Samano/Secretario.

fdo. Ldo. Gutierrez y Velasquez.- fdo. Ldo. Gregorio Lopez.- (fdo) I.do. Salmeron.

ILUSTRACIONES: Carlos V.- Armas del Reino de Castilla y Leon concedidas a don Juan Tito Túpac Amaru por Carlos I en 1-X-544 para su familia y descendientes.

NUMERO 7:/ REAL CEDULA/ Expedida por la Majestad del Sor. Dn. Carlos / $5^{\circ}$ en Valladolid a 9de Mayo de 1545 / A FABOR De Dn. Juan Tito (2 ${ }^{\text {a) }}$ Tupac-Amaro Inga, hijo/lexitimo de Tupac-Amaro, Sobrino de/Cusitito, y de Manco Inga Señores del Perú/ POR LA QUE/ Su Magestad señaló el escudo de Armas/ y Blasones de que debía usart dicho Dn. Juan/ y todos, y cada uno de sus Descendientes en / sus casas, Reporteros, y demás ptes. qe, quisiesen". (f. 128-130). 545.

ILUSTRÁCIONES: Ármas concedidas por Carlos I a Juan Tito Túpac Amaru en 9.V.

NUMERO 8, REAL CEDULA / Expedida en Valaldolid á 21 de Julio de 1553/ Por la que La cesaría Majestad del Sr. dn. Carlos 5\%/ y Da. Juana madre hizieron Merced a / Dn. Martín Hurtado de Arvieto Conquis/tador, y Pacificador del Perú/ De/El oficio de Rexidor perpetuó de la Ciudad/ del Cuzco, en atención a sus meritos, y servos. / el que usó, $y$ exerció, desde el 25 de Noviombre de 1554 segun Consta de las Diligencias insertas, en el / Testimonio dado en 7 de Agesto de 780 por Dn./ Juan Bapta. Gamarra essno. del Ayuntamiento del/Cuzco. (f. 131-135).

NUMERO @/ INFORMACION / Dada á Pedimento de la Ylte. Sa. Da. María Manrrique/. Coya Cusiguarcay vezina de esta Ymperial, gran Ciudad del Cizco/Cabeza de estos Reynos, y Provincias del Perú./ ANTE/ La Real Justicia de ella en 13 de Fleno. de 156?./ Por la qual/ Consta que la susodicha fué hija lexima. de Mango Inga Yupanqui Rey. Sor. natural que/ fué de estos Dominios, y de Da. Cathalina Táype, y Chilque una de sus Mujeres, que. en primeras Nun-/zia's (sic) lo fué de Guascar Ynga su hermano según sus Rilos Gentilicos. Que la da. Maria se casó con Dn. Die-/go de Mendoza Cayretopa su hermano, tambien hijo de Mango, qe. uno, y otra fuerón Nielos de Guay-/na Capac, y Visnielos de Topa Ynga Yupanqui ssres. Nturales de estos Reynos q.e los governaron con los demas/ que iefiere: Que Mango su Pe. salió de Paz á recibir a los Conquistadores 8 leguas de esta Ciud.. junto a la/ cuesta de Vilcaconga, y los auxilio, y metió en ella con sus Yndios de Guerra, y entrego la Casa del Sol/ con todo su infinito Te-

(2a) La Real cédula de 9-V-1545, dada en Valladolid, fué otorgada a don Crístóbal Topa Inga, hijo de Huayna Cápac y hermano de Atahualpa, señalándole escudo de armas para sí $Y$ sus descendientes" (MANUSCRITOS EN LAS BIBLIOTECAS Y ARCHIVOS DE EUOPA Y AMERICA por R. Vargas Ugarte, Tomo V, Suplemento.- Buenos Aires, 1947. p. 210-212. 
soro, que lomada posesión por los Reyes de Castilla de esta Ciudad, se coronó a Mango/ y dió la obediencia. Que inmedialamente apresó los espias, y emisarios que Chalcuchima Capn. de A!a-/gualpa embio al Quizquiz su comp. que ocupava el Cuzco, y su tierra para que aniquilase a los españoles por ser/ mortales, y combencido Chalcuchima de esta traición se le quemó for Pizarro on la Plaza de Xaquija/quana. Que osligado Mango de las instancias de Pizarro sobre la manílestación de Tesoros, y malos Iratamientos/ de su persona se retiró á Vilcabamba donde le mató cierlo Español. Que doña Maria vivia pobremente sin la de/sencia, y porle debido a su nacimiento; Que de los $3 \mathrm{mil}$ pesos que se la dieron por el Sor. Marques de Cañele para alimentos sobre los/ Repartimientos de los Indios de su HIZA? la quitarón 2 mil que no obstante cuidaba a su hija lejitima, Da. Beatriz, que/ estaba en Sta. Clara. Que el no reducirse D. Diego Cusilito Yupangui (de quien procedierón los ssres. Marqueses/de Ooropesa y Alcañizas) su hermano hera por la miseria en que estaba su hermana. Que como a Sra. nalural/de este Rno. la obsdecien lodos los Yndios del, aun los de Guerra de los que muchos se redujerón por sus insinuaciones.

GUIA YNFORMACION/ Se amplió en 15 de Enero de 1580 á Pedimento de Juan Fernandez Coronel Marido en segundas numcias (sic) de dicha/Da. Maria, haziendo conslat sus distinguidos merilos de uno, y otro, y que Mango despues que entregó esla Ciudad/ y Templo del Sol, derrolo al Quizquiz que habia alborotado la tierra, lo redujo a la obediencia de S. M. lodos los Yndios, y a los de Chinchaysuio, que en el valle de Jauja tenían cercado al tesorero Riqualme con otros españoles que conducian / el lesoro de S .M., y los persiguió, hasta que unos, y oiros estubieron en salbamento, y la tierra sosegada, que aunque Pizarro/ lo prometió dar la 3a. de los Indios, y tierra del Rejno por sus sucesores, no le dió nada, y la maltrató por que no le daba los tesoros/ de sus pasados, por lc que se retiró a Vilcabamba con su hijo Tunât-Amoro que era su subcesor/ Que en atencion a lodo seria / combeniente que para descargar S. M. su Real Conciencia mandase fundarles un Mayorazgo con $6 \mathrm{mil}$ Yndios de encomienda/perpelua para ellos, su hija doña Nelchora de Sclomayor, y sus subcesores como en los Mayorazgos de Castilla, y/ademas contiene algunas noticias guslosas. (f. 136-185).

\section{TESTIGOS DE ESTA YNFORMACIONA(f.Ci37) lli ConverSO»}

\section{NOMBRES.}

Dn. Cristobal linenez, Provisor del Cuzco $\ldots \ldots \ldots \ldots$

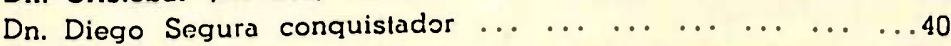

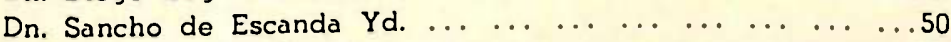

Dn. Diego de Trujillo Yd. ... . . . . . . . . . . . . . . . . 60

Dn. Juan de Pancorbo Yd. ... $\ldots . \ldots \ldots \ldots$

Dn. Mansio de Sierra Yd. ...

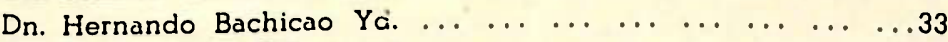

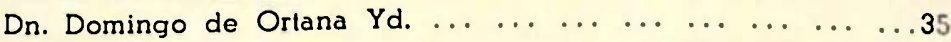

declaró 2 veces.

. on García Quispe Guara orexon ... . . . . . . . . . . . .73

Dn. Hernando Guerra conquistador ... ... ... . . . . . .40

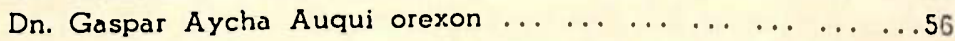


NOMBRES.

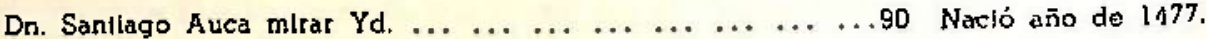

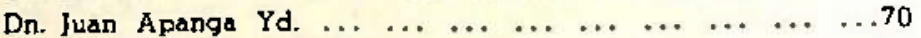

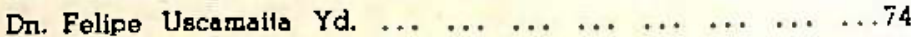

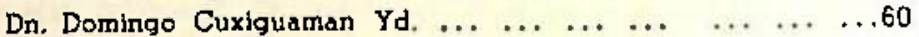

\section{OREXONES}

Dn. Felipe Caritipa. Cuñado de Mango porque en su hermana culo nombre calle dlce ques tuvo á Topac Amaro ... . . .70

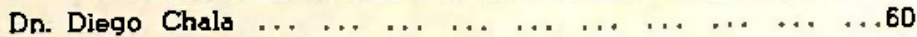

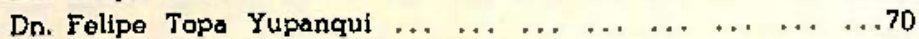

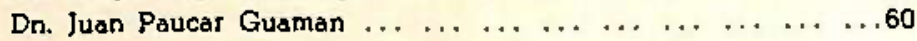

\section{CONQUISTADORES}

Dn. Juan Albatez Maldonado ..................40

Dn. Peord de Orne ... ... ... ...

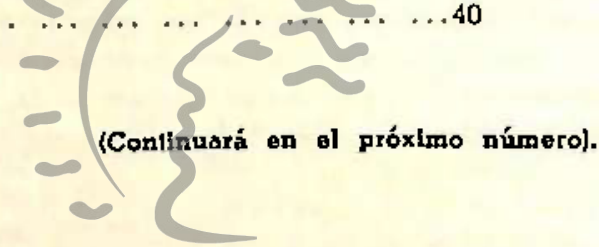

Biblioteca de Letras "Jorge Puccimetti Converso» 